\title{
THE RELATIONSHIP BETWEEN SOME ANTHROPOLOGICAL FACTORS AND SPORTS PERFORMANCE
}

\author{
I. MALARECKI, M.D. \\ Department of Physiology, Academy of Physical Education, \\ Marymoncka 34, Warsaw 45, Poland
}

The enormous working capacity of little animals like ants and the much lower physical performance of higher animals as well as men, in relation to their body size and weight, is well known. This is a natural biological phenomenon because the strength of the muscle is the square function, and body weight is the cubic function of linear size. Thus the body mass overtakes the strength of muscles with increasing the body size and of course body weight. Therefore it is interesting to know if there is any influence of human body size and weight on the sports performance.

It is established and well known fact that the relative strength i.e. maximal strength $\left(F_{\max }\right)$ divided by body weight $\left(B_{w} 1\right)$ is lower in subjects of higher body weight than in lighter ones.

This fact may be observed at the athletes of different weight in such kind of sports as weight lifting (fig. 1)

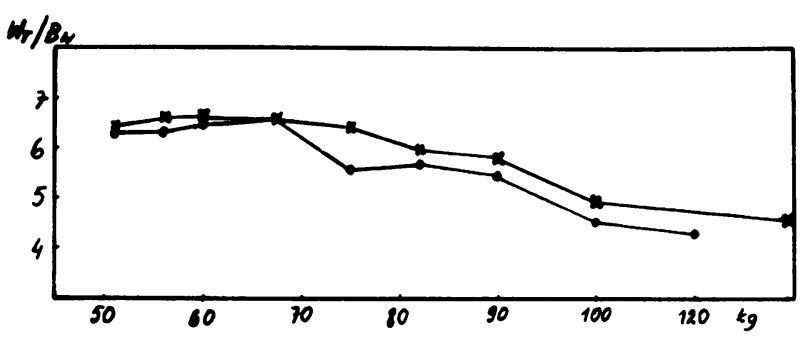

Fig. 1 Relation between relative strength and all weight classes.

WT woight-lifting records; $x$ world, $\bullet$ country

$B_{w}$ body weight class

Analysis of relation of body weight and strength is more clearly stated by the following procedure: Let us imagine human body as a cubic shape, thus the body weight $\left(B_{w}\right)$ is the cubic function of linear size, and the strength is proportionate to the cross section of the muscle. In our case cross section is equal to $\left(\sqrt[3]{B_{w}}\right)^{2}$.

If we divide total weight lifted $\left(W_{T}\right)$ by $\left(\sqrt[3]{B_{W}}\right)^{2}$ the results will represent weight lifted on cross section unit, which allows one to compare more objectively the strength in relation to weight.

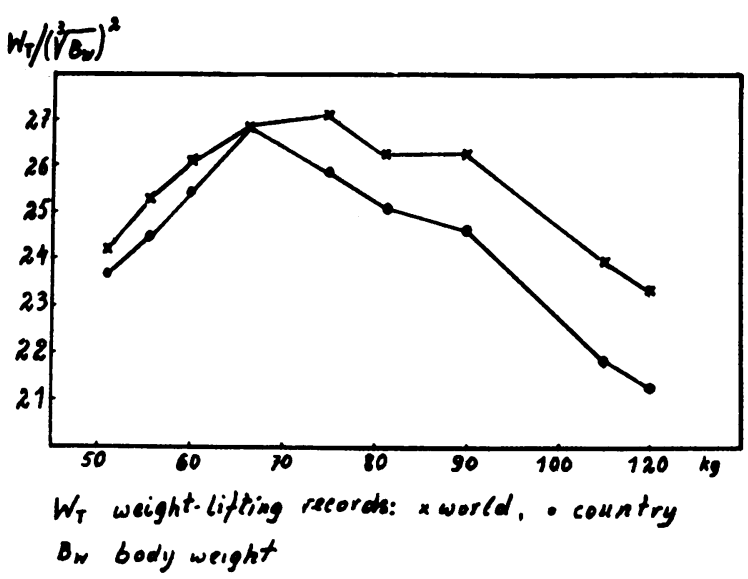

Fig. 2. Relation between weight lifted on cross section unit and all woight classes.

$W_{T}$ woight-lifting records; $x$ world, $\bullet$ country

$B_{w}$ body woight

We have also noticed that the maximal oxygen consumption $\left(\mathrm{V}_{\mathrm{O}_{2}}\right.$ max) with subjects such as boxers of heavier body weight tends to decrease under the same training conditions (Fig. 3)

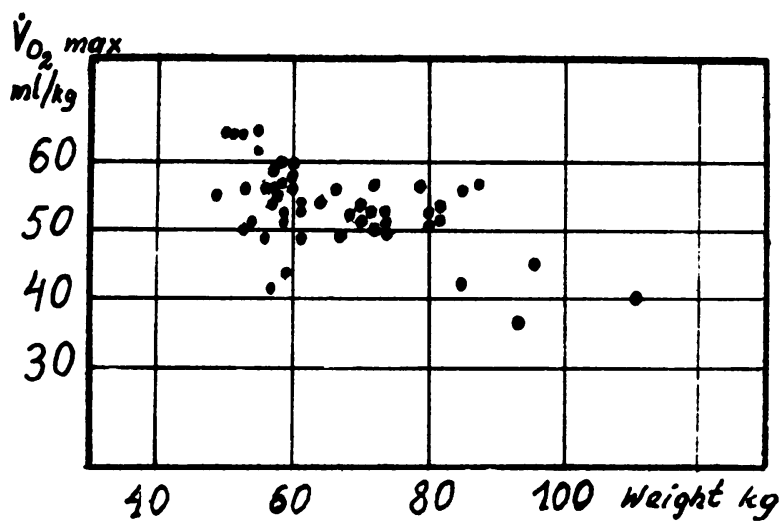

Fig. 3. Maximal oxygen consumption of subjects of different body woight

In view of observations mentioned it was interesting to explain the effectiveness of physiological functions in 
relation to body size and weight. In order to explain this fact we have managed to carry out a few measurements of maximal cardiac output per $\mathrm{m}^{2}$ of surface $\left(Q_{\max } / \mathrm{m}^{2}\right)$ in subjects having different height, but the $\mathrm{CO}_{2}$ rebreathing method. Students of physical education served as subjects.

Though the results presented in fig. 4 are very dispersed one can notice that the general condensed line of results decreases from shorter to taller height. That might be one of the factors limiting the possibility of oxygen uptake $\dot{\mathrm{V}}_{2} \max$.

It is obvious that these results presented as a preliminary paper should be confirmed on a larger number of human subjects. We consider the problem of body size and its influence on physiological functions and sports performance as significant from the theoretical and practical point of view, especially with relation to the observed tendency of average height increase which has been reported recently in many countries.

$\dot{Q}_{\max } \mathrm{l} / \mathrm{m}^{2}$

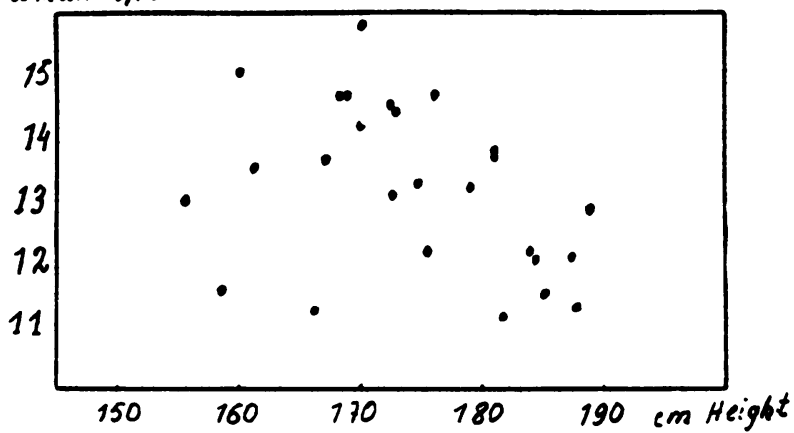

Fig. 4. Maximal cardiac output $\div m^{2}$ of subjects of different body height. 